\title{
EDITORIAL
}

\section{Extubation Algorithms: Why, where and when do we need them?}

\author{
Stephanie Whitener and Ulrich Schmidt*
}

Department of Anesthesia, Critical Care and Pain Medicine, Massachusetts General Hospital, Boston, MA, USA

\section{EXTUBATION}

Algorithms are becoming increasingly popular as a standardized approach to difficult and unique problems. Many resources have been spent on searching for a perfect algorithm for difficult intubations. In this issue, Voscopoulos et al. have proposed in this article an algorithm of extubation in the operating room after a difficult intubation. In this editorial we will first concentrate on the need for an OR post extubation algorithm and secondly, we will discuss the merits of its steps as well as present further recommendations.

An algorithm is particularly helpful, when it can be applied specifically to a common problem with common pathophysiologic abnormalities. The incidence of reintubation in the post-anesthesia care unit after elective surgical procedures has been reported as low, making this a rare event [1]. It might be that this algorithm for extubation may be better applied to areas in which the reintubation rate has been demonstrated to be considerably higher, such as the ICU. Reintubation in critically ill patients has been demonstrated to be as high as $25-30 \%$, in contrast only $5 \%$ of cardiothoracic, general surgical and trauma patients ultimately require reintubation, although this number increases with comorbid conditions [2].

The algorithm begins with multiple steps to assess the patient for extubation including palpating the cricoid membrane, ensuring a nearby expert in emergency cricothyroidotomy, reassessment of airway with laryngoscopy and possible consideration of peri-operative corticosteroids. Assessing the patient for extubation, may in fact be the most important component of this difficult extubation algorithm. Reassessment of the airway in the perioperative period can be very valuable in a patient that required large volume resuscitation or at high risk for laryngeal edema, but this assessment should determine whether the patient is suitable for extubation. Information from this laryngoscopy can be related to the critical care team when the decision is made to leave the patient intubated and assist them in evaluating when it will be safe for extubation. Recent data has shown that the use of video laryngoscopy might be especially valuable to assess the airway prior to extubation [3].

\footnotetext{
*Address correspondence to this author at the Surgical Intensive Care Unit, Massachusetts General Hospital, Department of Anesthesia, Critical Care and Pain Medicine, White 4, 537, Boston, MA 02114, USA;

Tel: 617-726-2859; Fax: 617-724-8511; E-mail: uschmidt@partners.org
}

The use of corticosteroids in the perioperative period to reduce the likelihood of reintubation secondary to laryngeal edema is suggested in this algorithm prior to extubation. A randomized control trial showed the efficacy of corticosteroids given prior to extubation in decreasing reintubation. However this trial was done in critically ill patients with prolonged intubation and showed that corticosteroids are probably more effective if given 12 hours prior to extubation. This data does not seem generalizable to the extubation of patients in the operating room following elective surgical procedures [4]. Steroids have some serious side effects and should not be considered in many patient populations, particularly when there is already such a low incidence of reintubation.

Once the decision to extubate has been made, the algorithm's first branch point is deciding to use an airway exchange catheter or a gum elastic bougie. The authors conclude that anesthesiologists may not have access to an airway exchange catheter and that a bougie may be more accessible and therefore a good alternative. The problem with the use of a bougie in this algorithm is that it is suggested in place of a soft catheter that can be used to administer supplemental oxygen. A bougie is significantly more rigid which increases the possibility of airway injury. Indeed, severe complications such as pharyngeal wall perforation have been reported with its use [5]. Furthermore, patients may demonstrate an inability to tolerate it for the time duration deemed necessary without the benefit of being able to ventilate through it. A rigid bougie makes it difficult to assist the patient with mask ventilation potentially creating a problem with oxygenation prior to reintubation, making this branch of the algorithm more difficult to implement.

Once the airway has been instrumented with either an AEC or a GEB, the device is to be left in place until the patient has proven that they have adequate oxygenation and ventilation. The authors suggest that this may be 30-60 minutes or until the likelihood of reintubation is minimized. Given the pressure for operating room efficiency, the patient will most likely be brought to the post anesthesia care unit or ICU with the device in place. The patient must tolerate the device during this time period, in which they will be transferred off of the operating room table onto a stretcher and out of the operating room allowing for multiple opportunities for malposition or dislodgement of the device. Since the anesthesiologist who originally intubated the patient is usually not available in the PACU or the ICU, 


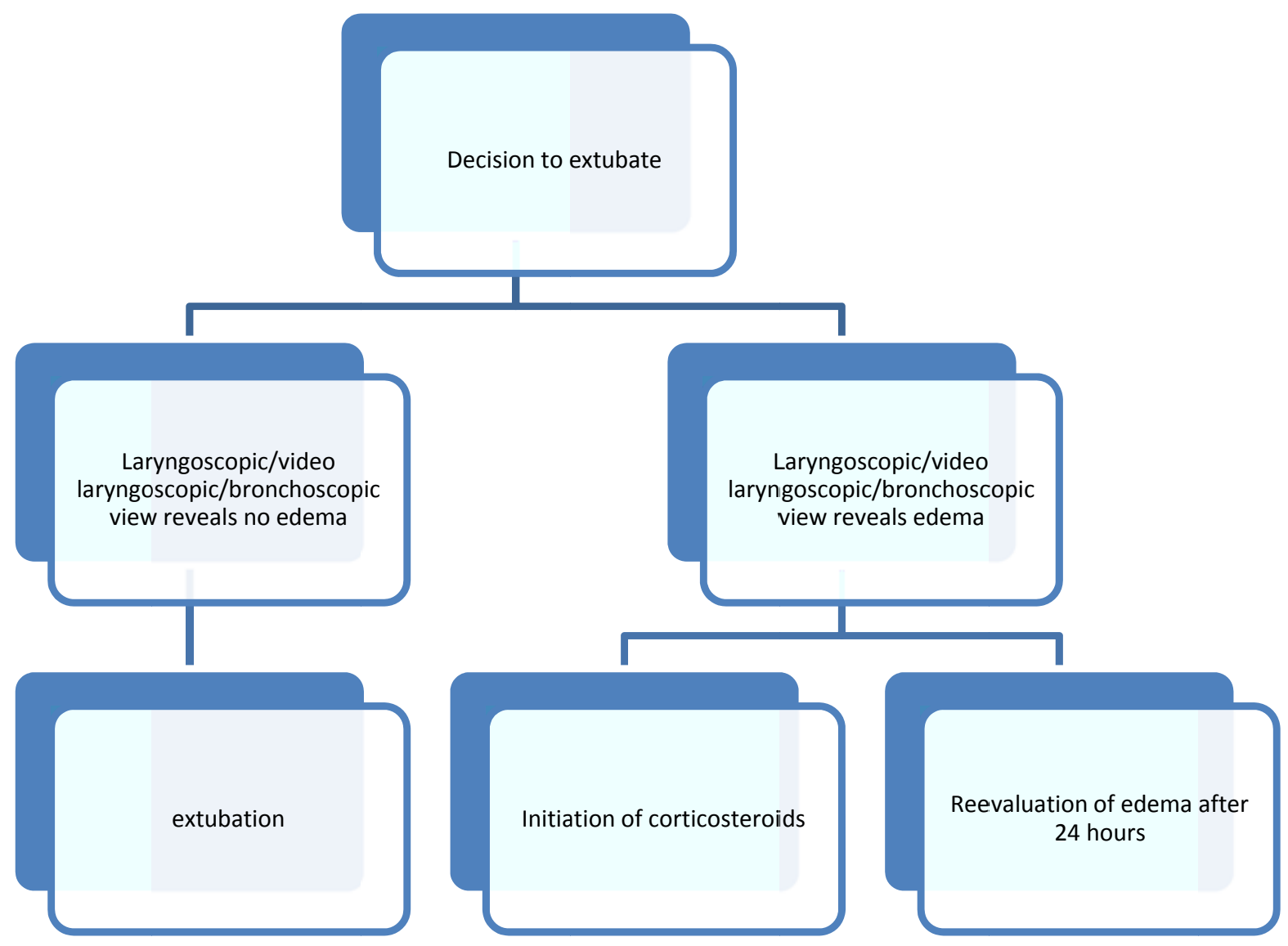

Fig. (1). Extubation algorithm.

another provider will determine when it is safe to remove the device.

A difficult to intubate patient poses a unique problem during extubation and the authors should be commended for proposing an algorithm to ensure the safety of these patients. Given current available information, we would recommend slightly modifying the algorithm (Fig. 1). After a decision to extubate is made the airway should be examined for the existence of edema. If no edema is present the patient should be extubated. If significant edema is present the patient should stay intubated and initiation of steroids considered. The patient should be reevaluated for extubation after $24 \mathrm{~h}$.

We encourage the wider community to undertake validation of an extubation algorithm. Furthermore, given the high incidence of reintubation in ICU patients, an ICU extubation algorithm seems important.

\section{CONFLICT OF INTEREST}

\section{ACKNOWLEDGEMENT}

Declared none.

\section{REFERENCES}

[1] Meng L, Quinlan J, Underwood J. Incidence and morbidity of reintubation after general anesthesia in the post anesthesia care unit. Anesthesiology 2004; 101: A1564.

[2] Demling RH, Read T, Lind LJ et al. Incidence and morbidity of extubation failure in surgical intensive care patients. Crit Care Med 1988, 16: 573-7.

[3] Newmark JL, Ahn YK, Adams MC et al. Use of video laryngoscopy and camera phones to communicate progression of laryngeal edema in assessing for extubation: a case series. J Intensive Care Med 2012, [Epub ahead of print].

[4] McCaffery J, Farrell C, Whiting P, Dan A, Bagshaw SM, Delaney AP. Corticosteroids to prevent extubation failure: a systematic review and meta-analysis. Intensive Care Med 2009, 35: 977-86.

[5] Kadry M , Popat M. Pharyngeal wall perforation-an unusual complication of blind intubation with a gum elastic bougie. Anesthesia 1999; 54: 404-5.

Declared none. 